\title{
A special issue on selected papers from the 5th Hamlyn Symposium on Medical Robotics, 2012
}

\author{
Guang-Zhong Yang $\cdot$ Karen Kerr $\cdot$ Ara Darzi
}

Published online: 4 August 2013

(C) Springer-Verlag London 2013

The Hamlyn Symposium is an annual international forum for clinicians, engineers and researchers to exchange ideas and explore new challenges and opportunities in medical robotics and allied technologies. The 5th Symposium was another success, due to the range of papers presented from leading international teams, and the growing workshop series, which is organised by teams of senior academics and young research fellows to further stimulate academic networking and collaboration.

For this special issue, we have selected five representative manuscripts from the 52 papers from 11 countries presented at the Symposium, based on the initial review scores and the quality of the presentation during the meeting. They represent the unique multidisciplinary blend of the Symposium and our increasing focus on imaging and navigation, and usability by the surgeon to improve patient outcomes through technological innovation.

The selected papers focus on novel developments in visualisation and navigation, and explore usability and skills assessment of new techniques. Visualisation and imaging are both important factors to consider in robotic surgery. The development and use of video augmentation for intraoperative image guidance for transoral robotic surgery is described by Liu et al. Using preoperative planning data, the team have demonstrated that the data can be registered to the intraoperative endoscopic view to augment and define targets, which will improve the operation through improved navigation, orientation and ultimately tumour resection. This has been piloted in situ to show improvements over and above simulated standard practice. Lee et al. explore improvements in navigation in vascular surgery, proposing a novel motionadaptive catheter navigation scheme to compensate for

G.-Z. Yang $\cdot$ K. Kerr $(\bowtie) \cdot$ A. Darzi

The Hamlyn Centre, Imperial College London, London, UK

e-mail: k.kerr@imperial.ac.uk physiological motion and tortuous vessels. A simulated aortic branch cannulation procedure was used to demonstrate improved visualisation through dynamic shape modelling and real-time instantiation in conjunction with a reconstructed view of the catheter tip to enhance the usability for the surgeon.

Improvements in efficiency and usability of robotic systems are increasingly being explored. In the paper by Simaan et al., a robotic system for single port access undergoes preliminary evaluation, demonstrating its design and functionality for basic tasks in minimally invasive surgery, manipulation, knot tying and visualisation/tracking. Ruiter et al. describe a novel robotic system that can be appended to a traditional flexible endoscope for advanced procedures, in particular those that need a team for complex and enhanced manoeuvrability. Initial results indicate that the robotic system reduces the time required to complete the investigation and that usability is enhanced from the physician's perspective. Given the increased use of robotic systems, it is important to monitor and assess the surgeon's skills as well as the technical developments. This is highlighted in the paper by Jun et al., which explores the use of a quantitative computer-based surgical performance evaluation scheme for assessing surgeons of varying levels.

The Symposium would not be possible without the commitment and hard work of the programme and organising committees. We are also very grateful to all our distinguished invited speakers, particularly Professor Shigeo Hirose from Tokyo Institute of Technology, who provided an overview of "Biologically Inspired Robots: Snake-like, Spider-like Robots and Others", and Professor Richard Satava, who gave the inaugural Karl Storz-Harold Hopkins lecture on "Developing Next Generation Robots" that stimulated the panel debate.

Conflict of interest None disclosed. 